DOI: $10.12731 / 2306-1561-2013-4-25$

\title{
IDENTIFICATION OF RESOURCES AND ACTIVITIES IN COMPLEX DYNAMIC SYSTEMS
}

Borshch V.V., Prikhodko L.V., Prikhodko M.V.

\begin{abstract}
The article deals with the modeling of complex dynamic systems. . A classification of types of resources for permanent and temporary. It is shown that for regular resources are the typical parameters that identify them in the health and well-established to perform certain actions. In addition, each action is related to the change of the dynamic state of a complex system. Changing its state is set event to be formally defined and fixed in the model. In this case, unlike regular events, irregular wear a pronounced stochastic in nature and not preplanned management system. It is shown that their appearance may violate the synchronization of regular events and result in them being early or normal completion.
\end{abstract}

Keywords: a complex dynamic system, system resources, regular events, irregular events, the relevance of action, productional grammar.

\section{УДК 681.3}

\section{ИДЕНТИФИКАЦИЯ РЕСУРСОВ И ДЕЙСТВИЙ В СЛОЖНОЙ ДИНАМИЧЕСКОЙ СИСТЕМЕ}

\section{Борщ В.В., Приходько Л.В., Приходько М.В.}

\section{Аннотация}

В статье рассматриваются вопросы моделирования сложных динамических систем. Дается классификация видов ресурсов на постоянные и временные. Показано, что для постоянных ресурсов характерными являются параметры, идентифицирующие их по работоспособности и налаженности на выполнение определенных действий. При этом каждое действие связано с изменением состояния сложной динамической системы. Изменение ее состояния представляет совокупность событие, которые должны быть формально определены и зафиксированы в модели. При этом в отличие от регулярных событий, нерегулярные носят ярко выраженный стохастический характер и заранее не планируются системой управления. Показано, что их возникновение может нарушать синхронизацию регулярных событий и приводить к невозможности их начала или нормального завершения. 
Ключевые слова: сложная динамическая система, ресурсы системы, регулярные события, нерегулярные события, релевантность действий, продукционная грамматика.

Практически любой объект управления обладает большим количеством свойств и поэтому изучить его полностью практически невозможно [11 - 15]. Следовательно, необходимо определить ограниченное множество характеристик, описывающих конкретный объект наилучшим образом. Тогда объект представляется как модель существенных сторон предметной области и идентифицируется в соответствии с анализируемой проблемой или задачей. Он задается набором свойств и назначением каждому из этих свойств переменной.

Сложную динамическую систему (СДС) на концептуальном уровне представим как множество некоторых ресурсов, взаимодействующих между собой [1]. Ресурс - это элемент СДС, внутренней структурой которого можно пренебречь, в то время как наличие и свойства его как целого важны для целей описания. В зависимости от поставленной задачи ресурсом может быть любая часть системы.

Примерами ресурсов в СДС являются человек, оборудование, транспортные средства, группа людей, партии изделий, склады и другие физически существующие объекты рассматриваемой СДС. Помимо указанных могут вводиться в рассмотрение в качестве ресурсов различного рода абстракции, такие как система, роли, пространство состояний и т.п.

Каждый ресурс в модели СДС должен получить уникальное имя, отличающего его от других ресурсов. Имя желательно давать ресурсу таким образом, чтобы оно идентифицировало его назначение и взаимосвязь с другими ресурсами. Это связано с тем, что второе имя в большей степени отражает специфику именно моделируемого объекта, а не любого объекта с очередью. Если ресурсов много, то имеет смысл в имя ввести порядковые номера. Помимо получения имени каждый ресурс СДС в модели должен быть описан.

Ресурсы могут быть двух видов: постоянные и временные. Постоянные ресурсы всегда присутствуют в системе (порт, бензоколонка, склад, транспортные устройства, рабочий и т.п.). Временные ресурсы поступают в СДС и покидают ее в процессе функционирования, они могут быть результатом работы СДС [2].

Все ресурсы СДС образуют некоторое множество:

$R=\left\{r_{i} / i=1, \ldots, N(t)\right\}$,

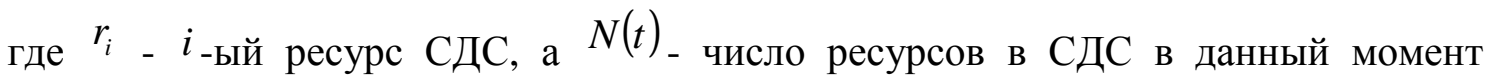
времени.

Каждый ресурс описывается множеством его параметров. Параметры могут быть трех различных типов:

- описательные, представляющие факты, внутренне присущие каждому ресурсу;

- указывающие, используемые для дачи имени или обозначения ресурса. Они часто используются как идентификаторы ресурсов; 
- вспомогательные, используемые для связи различных ресурсов, накопления статистики, графического вывода при имитации и т.п.

Часть параметров ресурса изменяется в процессе функционирования СДС, а другие остаются постоянными.

Для постоянных ресурсов характерными являются параметры, идентифицирующие их по работоспособности и налаженности на выполнение определенных действий. Для временных ресурсов важными являются параметры, описывающие месторасположение, участие в некоторых действиях, частота появления, степень изменения ресурса и ряд других.

Множество ресурсов можно разбить на несколько непересекающихся подмножеств однотипных ресурсов $R_{k} \subseteq R, k=1,2, \ldots, K$. Все ресурсы одного типа имеют одни и те же параметры и что особенно важно ведут себя одинаковым образом в одинаковых условиях. Каждый ресурс модели СДС должен быть отнесен к какомулибо типу:

$$
R=\bigcup_{k} R_{k} \bigcap_{k} R_{k}=\varnothing .
$$

Состояние і-го ресурса $C_{i}(t)$, в момент времени $t$ будем описывать значениями его параметров:

$$
C_{i}(t)=\left\{c_{i j}(t) / j=1, \ldots, M_{i}\right\}
$$

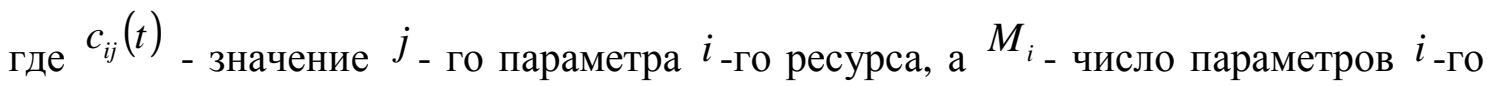
pecypca.

Тогда состояние СДС будем описывать множеством состояний всех ее ресурсов:

$$
C(t)=\left\{C_{i}(t) / i=1, \ldots, N\right\} \text {. }
$$

Ресурсы, принадлежащие к одному типу, наследуют общие свойства этого типа. Отношения наследования может использоваться как для отражения общности ресурсов типа, так и для идентификации ассоциативных связей [3].

Ресурсы взаимодействуют друг с другом в соответствии с определенными закономерностями, выполняя различные действия. Каждое действие связано с изменением состояния СДС. Изменение состояния СДС называют событием. Все события должны быть как-то определены и зафиксированы в модели.

Событие в модели это - абстракция инцидента или сигнала в реальном мире, который сообщает о переходе каких-либо объектов в новое состояние. События могут быть внешними по отношению к СДС и принадлежать ей самой. То есть значение события заключается в некоторой фразе, несущей информацию о том, что произошло в СДС или о том, на что СДС должна реагировать.

События в дискретных системах происходят в счетные моменты времени, которые должны фиксироваться в модели с помощью независимой переменной, в качестве которой чаще всего принимается модельное (системное) время [4]. Эта переменная изменяется дискретно и служит базой для определения различий в наблюдении одного и того же свойства СДС. 
Все события каким-либо образом влияют на состояние СДС и это влияние должно быть зафиксировано в модели. Поэтому событие можно рассматривать как сигнал, передающий данные (управление) об определенном состоянии СДС для некоторого действия, которое может либо начаться, либо завершиться (события начала и окончания). Следовательно, можно дать еще одно определение события, определив его как неделимое элементарное действие, в том смысле, что оно не имеет протяженности во времени.

Все события делятся на регулярные и нерегулярные.

Регулярными называют события, вызываемые штатным функционированием ресурсов [5]. Изменения в системе при совершении регулярного события можно формализовать, поскольку они отражают логику взаимодействия ресурсов между собой. Регулярные события могут быть частично упорядочены во времени, то есть для них может быть построена временная диаграмма.

Регулярное событие формально представим как некоторое изменение состояния системы и опишем следующим образом:

$$
e=\left(t_{e}, C_{e}^{-}, C_{e}^{+}\right)
$$

где $t_{e}$ - момент времени свершения события $e, C_{e}^{-}$- состояние СДС до события $e$, ${ }_{\mathrm{a}} C_{e}^{+}$- состояние СДС после события $e$.

Так как состояние дискретной системы изменяется только в моменты свершения событий, то оно остается неизменным между событием $e_{i}$ и событием $e_{i+1}$, и, следовательно, имеет место равенство:

$$
C_{e_{i}}^{+}=C_{e_{i+1}}^{-} .
$$

Очевидно, что в некотором событии е участвуют не все ресурсы системы, и его наступление приводит лишь к локальному изменению состояния системы. Ресурсы, состояние которых изменяется, называют релевантными событию е:

$$
\begin{aligned}
& R_{e}=\left\{r_{i e} / C_{i}^{-}\left(t_{e}\right) \neq C_{i}^{+}\left(t_{e}\right)\right\}, \\
& \text { где } r_{i e}-i \text {-ый ресурс СДС, релевантный событию } e, \text { и } R_{e} \subseteq R
\end{aligned}
$$

Выделение ресурсов релевантных событию достаточно важный этап описания СДС, так как он некоторым образом формализует взаимодействие ресурсов СДС между собой, отражая структурную и динамическую связность СДС. Выделив релевантные событию ресурсы, мы получаем возможность при моделировании события рассматривать изменения состояния не всей модели СДС, а лишь некоторой локальной, часто весьма ограниченной ее части. Это упрощает моделирование, уменьшает объем обрабатываемых данных, делает описание знаний компактным и ясным.

Нерегулярные события [6] происходят либо при нештатной работе ресурсов (поломки, отказы), либо из-за внешних по отношению к системе причин (приход в систему нового временного ресурса, изменение состава постоянных ресурсов при модификации системы и т.п.). В отличие от регулярных событий, нерегулярные носят ярко выраженный стохастический характер и заранее не планируются системой 
управления. Их возникновение может нарушать синхронизацию регулярных событий, приводить к невозможности их начала или нормального завершения.

Для нерегулярного события $\tilde{e}$ состояние ресурсов до его начала $C_{\tilde{e}}^{-}$ непредсказуемо. Так как $\widetilde{e}$ происходит случайным образом, то оно описывается лишь временем возникновения и состоянием ресурсов после нерегулярного события:

$$
\tilde{e}=\left(t_{\tilde{e}}, C_{\tilde{e}}^{+}\right),
$$

где $t_{\tilde{e}}$ - время наступления нерегулярного события, а $C_{\tilde{e}}^{+}$- состояние системы после наступления нерегулярного события.

При совершении событий каждый ресурс СДС меняет свое состояние. Изменение состояния некоторого ресурса может быть представлено как модель состояния, которая состоит из множества состояний и множества событий, переводящих ресурс из состояния в состояние.

Ресурсы одного типа меняют свое состояние согласно одним и тем же правилам. Поэтому модель состояний является абстракцией поведения всех однотипных ресурсов СДС и однозначно соответствует абстракции “Тип ресурса".

Модель состояний статичная по своей природе не обеспечивает процесс имитации необходимыми знаниями, но она полезна на определенном этапе описания СДС, так как позволяет верифицировать разбиение ресурсов на типы и идентифицировать множество событий в системе.

Введя абстракцию события, можно уточнить понятие дискретности системы, охарактеризовав ее двумя свойствами:

- состав СДС может быть описан счетным множеством ресурсов;

- изменения состояния СДС происходят в счетные моменты времени (события) и имеет место соотношение $C_{e_{i+1}}^{-}=C_{e_{i}}^{+}$.

Формализация событий и модели состояний является важным этапом перехода от СДС к ее модели, так как позволяет описать структурную и динамическую связность СДС, но не обеспечивает представления динамики процесса. Для устранения этого положения необходимо рассмотреть взаимодействие событий на множестве ресурсов СДС.

Ресурсы в процессе функционирования СДС выполняют определенные действия, взаимодействуя между собой. С каждым действием связано два события - его начала и окончания. Действие, как правило, представляет собой целенаправленное мероприятие, выполняемое под управлением некоторой подсистемы и направленное на достижение определенной цели. Поэтому действие планируется и может находиться в различных состояниях: запланировано, начато, окончено и прервано по какой-либо причине. При успешном окончании действия считаем, что поставленная цель достигнута.

В СДС присутствует, как правило, большое количество разнообразных действий. Поэтому число действий в СДС ограничено только временем рассмотрения системы.

Используя понятие события, целенаправленное действие изменения состояния системы можно описать через события начала и окончания действия: 


$$
a=\left\langle e_{H}, e_{K}\right\rangle=\left\langle t_{H}, C_{H}^{-}, C_{H}^{+}, t_{K}, C_{K}^{-}, C_{K}^{+}\right\rangle,
$$

где $C_{H}^{-}, C_{H}^{+}$- состояние системы перед и после наступления события $e_{H}$, соответственно; $C_{K}^{-}, C_{K}^{+}$- состояние системы перед и после наступления события $e_{K}$, соответственно; $t_{H}, t_{K}$ - время начала и конца действия.

По аналогии с ресурсами, релевантными событию, определим множество ресурсов $R^{a}$, релевантных действию а, как объединение множеств ресурсов, релевантных событиям начала и конца действия:

$$
\mathrm{R}^{a}=\mathrm{R}^{e_{H}} \cup \mathrm{R}^{e_{K}} .
$$

Длительность действия определяется как $\Delta t=t_{K}-t_{H} \geq 0$. Действие с нулевой длительностью представляет собой событие. Данное соотношение справедливо, если в течение выполнения действия оно не прерывалось нерегулярными событиями.

Если во время протекания действия а, т.е. на интервале $\left[t_{H}, t_{K}\right]$ произошло нерегулярное событие $\tilde{e}$, затрагивающее ресурсы действия а $\left(\mathrm{R}^{e^{i}} \cap \mathrm{R}^{a} \neq \varnothing\right.$ ), то в общем случае дальнейшее протекание действия может оказаться невозможным. В этом случае результатом действия будет не состояние $C_{K}^{+}$, а состояние $C_{\tilde{e}}^{+}$, определяемое нерегулярным событием $\tilde{e}$.

Процесс функционирования СДС можно представить как временную последовательность действий и нерегулярных событий:

$$
\Pi=\langle A, \tilde{E}, \succ\rangle,
$$

где $A$ - множество действий; $\tilde{E}$ - множество нерегулярных событий; $\succ$ отношение предшествования во времени.

Для регулярного события можно указать алгоритм $F$ преобразования $C^{-} \stackrel{F}{\longrightarrow} C^{+}$, который определяется закономерностями функционирования СДС. Поэтому действие а можно представить следующим образом:

$$
a=\left\langle F_{H}\left(C_{K}^{-}\left(R^{a}\right)\right), F_{K}\left(C_{K}^{-}\left(R^{a}\right)\right), t_{H}, t_{K}\right\rangle
$$

где $F_{H}, F_{K}$ - алгоритмы преобразования параметров, описывающих состояние ресурсов при событиях $e_{H}{ }_{\text {и }} e_{K} ; C_{H, K}^{-}\left(R^{a}\right)$ - состояние ресурсов, релевантных действию $a$, до событий начала и конца действия.

Действие $a$ может начаться, если значения параметров его релевантных ресурсов $C_{H}^{-}\left(R^{a}\right)$ отвечает некоторому условию. Условие начала действия можно представить как некоторое логическое выражение $P\left(R^{a}\right)$, принимающее значения ИСТИНА или ЛОЖЬ (TRUE, FALS), в зависимости от текущего состояния релевантных действию ресурсов. 
Действие привязано ко временной оси: начинается в момент $t_{H}$ и кончается в $t_{K}$. Если в описании действия исключить привязку к временной оси, оставив лишь длительность его выполнения $\Delta t$, то получим виртуальное действие (возможное) [7]. Виртуальное действие может начаться (но не обязательно начнется), если для множества $R^{v}$ релевантных ему ресурсов выполняется условие $P\left(R^{v}\right)=T R U E$. Таким образом, виртуальное действие можно представить как:

$$
v=\left\langle P\left(R^{v}\right), F_{H}\left(C_{H}^{-}\left(R^{v}\right)\right), F_{K}\left(C_{K}^{-}\left(R^{v}\right)\right), \Delta t\right\rangle .
$$

Виртуальное действие отражает (алгоритмы $F_{H}, F_{K}$ и условие $P\left(R^{v}\right)$ ) логику взаимовлияния ресурсов СДС в процессе функционирования. Всякий раз, когда состояние СДС удовлетворяет условию начала виртуального действия, может произойти действие $a$, соответствующее данному виртуальному и имеющее определенные времена $t_{H}, t_{K}$. То есть виртуальное действие описывает, что может произойти в СДС и при каких условиях, a действие - что произошло/происходит/произойдет и в какое время.

Приведенное выше описание действия позволяет формализовать законы функционирования системы для дальнейшего их использования в имитационных моделях (ИМ). Однако для этого имеется ряд препятствий. Во-первых система, являясь сложной, характеризуется чрезвычайно большим числом действий, отличающихся используемыми ресурсами, временем протекания и т.д. В процессе функционирования системы в ней появляются все новые и новые временные ресурсы и соответствующие действия. Во-вторых, одно и тоже действие может выполняться различными ресурсами (взаимозаменяемыми), что еще больше увеличивает размерность задачи.

Выходом из этого положения является констатация того факта, что множество виртуальных действий $V=\left\{v_{q} / q=1,2, \ldots, Q\right\}$, относящихся к определенной системе, может быть разбито на небольшое число подмножеств действий, имеющих одинаковую природу. Это подмножества, которые составляют, например, действия транспортировки, обслуживания, ремонта и т.д. Виртуальные действия, принадлежащие такому подмножеству $V_{j} \subseteq V$, имеют одинаковую логику взаимодействия ресурсов, и различаются лишь конкретными ресурсами в них участвующими. Подмножество $V_{j}$ описывается следующим образом:

$$
V_{j}=\left\langle\Psi, P\left(R^{v}\right), F_{H}\left(C_{H}^{-}\left(R^{v}\right)\right), F_{K}\left(C_{K}^{-}\left(R^{v}\right)\right), \varphi\left(C_{H}^{-}\left(R^{v}\right)\right)\right\rangle,
$$

где $\Psi$ - множество всех возможных множеств $R^{v}$ релевантных ресурсов, с использованием которых можно выполнить виртуальное действие $v \in V_{j}$, а $\varphi\left(C_{H}^{-}\left(R^{v}\right)\right)$ длительность выполнения виртуального действия, зависящая от состояния используемых виртуальных ресурсов СДС. 
Таким образом, принадлежность виртуальных действий к определенному типу означает, что для них $P\left(R^{v}\right), F_{H}\left(C_{H}^{-}\left(R^{v}\right)\right){ }_{\text {и }} F_{K}\left(C_{K}^{-}\left(R^{v}\right)\right)$ одинаковы, т.е. действия одного типа одинаковым образом меняют состояние релевантных ресурсов, требуют одинаковых условий начала по всем ресурсам, и отличаются лишь множествами $R^{v}$, используемых ресурсов и временем выполнения.

Для формального описания логики виртуальных действий, принадлежащих подмножеству $V_{j}$, введем понятие операции. Операция о есть формальное описание множества однотипных виртуальных действий:

$$
o=\left\langle E(\Psi), P\left(R^{o}\right), F_{H}\left(C_{H}^{-}\left(R^{o}\right)\right), F_{K}\left(C_{K}^{-}\left(R^{o}\right)\right), \varphi\left(C_{H}^{-}\left(R^{o}\right)\right)\right\rangle,
$$

где $E(\Psi)$ - описание множества $\Psi ; R^{o}$ - множество формальных ресурсов операции.

Операцию о в некотором смысле можно уподобить подпрограмме, в которой

$P\left(R^{o}\right), F_{H}\left(C_{H}^{-}\left(R^{o}\right)\right)$ и $F_{K}\left(C_{K}^{-}\left(R^{o}\right)\right)$ - условие выполнения и алгоритмы, описанные в формальных параметрах.

При задании фактических параметров получаем из операции виртуальное действие [8]. Для этого на место каждого формального ресурса $r_{i} \in R^{o}$ операции о необходимо подставить любой ресурс из некоторого непустого множества однотипных ресурсов.

Операция, следовательно, отражает логику взаимодействия ресурсов системы в процессе функционирования. Всякий раз, когда состояние системы соответствует $P\left(R^{o}\right)$, может происходить действие а, описываемое операцией о, с различными $t_{H}$ и $t_{\kappa}$. Операция описывает, как происходит действие или виртуальное действие и с какими множествами релевантных ресурсов, т.е. что может произойти в СДС при определенных условиях, а действие - что произошло, происходит, произойдет и в какое время.

Приведенное понятие операция может быть представлено как модифицированное правило продукции, введенное ранее:

ЕСЛИ(условие)

TO1(событие 1)

ЖДАТЬ $\left(\varphi\left(C_{H}^{-}\left(R^{o}\right)\right)\right.$

TO2(событие 2).

Структура продукционного имитатора представлена на рисунке (рисунок 1). Основными его элементами являются динамическая продукционная система и аппарат событий. Действия инициируются системой вывода, а нерегулярные события имитируются специальным блоком. При имитации состояние системы изменяется в соответствии с описанием нерегулярного события либо действия, которое началось или завершилось. После любого изменения состояния, т.е. при каждом событии, вызывается система вывода. Она просматривает в Б3 все операции и проверяет по 
предусловиям, могут ли они начаться. При нахождении таких операций инициируются события начала соответствующих действий. Продукционная система (БД, БЗ и система вывода), система имитации нерегулярных событий и аппарат ведения событий совместно осуществляют построение модели процесса. На основании анализа результатов имитации на этой модели вычисляются различные показатели функционирования системы. Система трассировки выводит подробную информацию о событиях в специальный файл, который затем обрабатывается для детального анализа процесса и представления информации в удобном виде. Система анимации позволяет отображать на экране во время моделирования поведение системы.

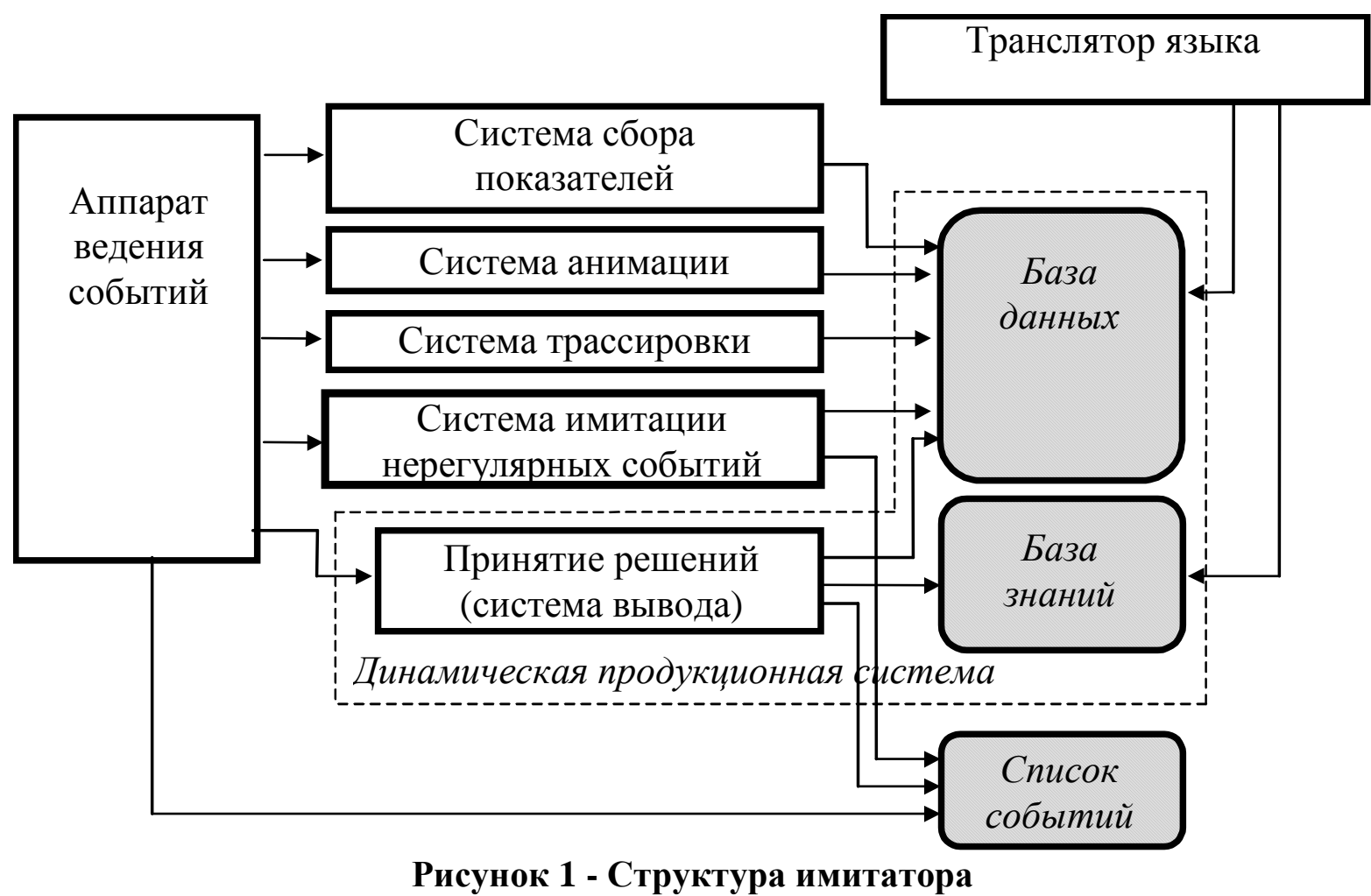

ИМ можно рассматривать как знания ее разработчиков об элементах СДС, их составе, структуре и о том, как они взаимодействуют между собой в процессе функционирования. ИМ сама по себе не позволяет получить законов функционирования СДС как системы и не раскрывает внутренние причинноследственные связи ее поведения. Она лишь позволяет получить значения выходных переменных по значениям входных.

Поэтому решение многих системных задач, связанных с анализом функционирования, синтезом структур, планированием, управлением и др. не обеспечивается чисто имитационным моделированием, так же как и чисто математическими методами. Как правило, для принятия решений используют сложное сочетание математики, вычислительных методов (обычно имитационного моделирования), эвристических подходов, экспериментальных методов и методов инженерии знаний (чаще всего экспертных систем). Комплексное использование 
указанных методов и средств обеспечивает пользователя поддержкой при принятии решений.

Необходимость сращивания имитационных моделей (ИМ) с методами исследования операций (оптимизацию) и искусственного интеллекта приводит к идее гибридных систем. Блок оптимизации реализует один из алгоритмов поисковой оптимизации (например, градиентный поиск), а ИМ служит для вычисления значений критерия оптимизации для выбираемых вариантов решения (рисунок 2).

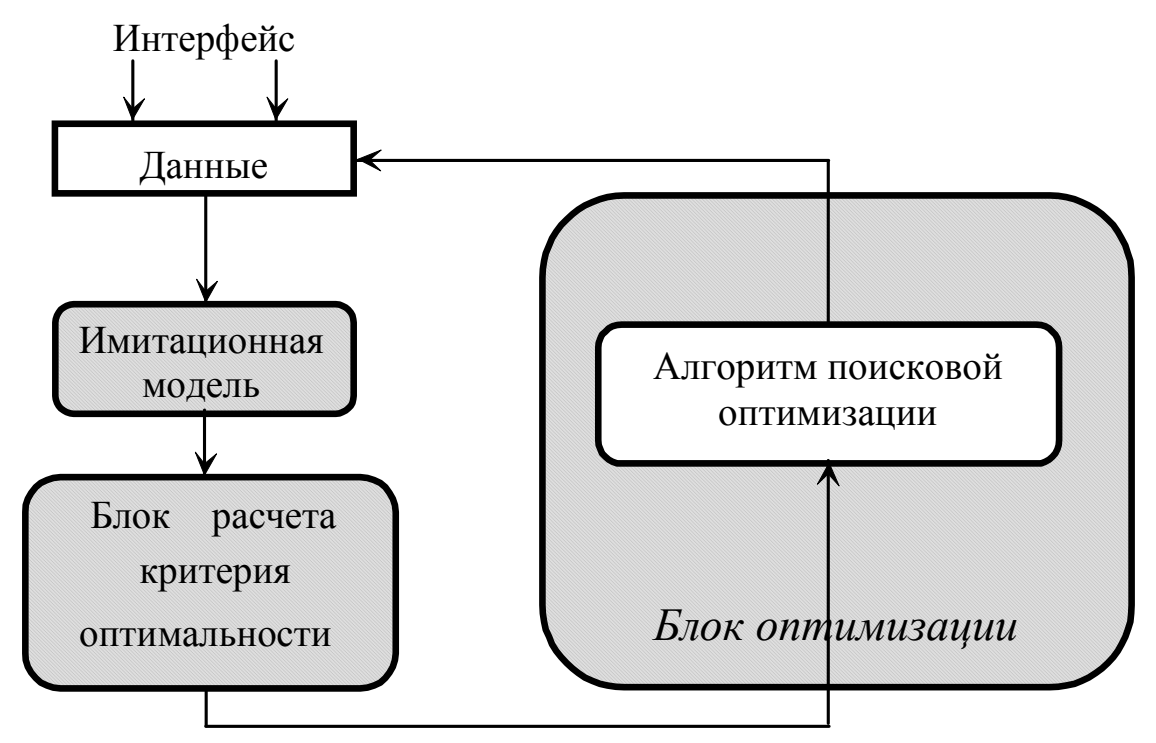

Рисунок 2 - Вариант гибридной системы

Прогон ИМ обеспечивает, в лучшем случае, получение достоверные результатов в одной точке пространства поиска решений. Проведение исследований при проектировании СДС требует реализации серии экспериментов в большой области поиска, целенаправленность которых обеспечивается в традиционных системах моделирования специалистом-разработчиком.

Для эффективного решения задач моделирование должно иметь интеллектуальную надстройку [9], позволяющую заменить специалиста-разработчика. Использование ИИ позволяет решить проблемы, связанные с накоплением и использованием знаний, их пополнением, выводом новых знаний на основе имеющихся в системе, повышением эффективности моделирования. Получение, обобщение и хранение знаний от специалистов-разработчиков может быть проведено с высоким качеством при использовании экспертных систем.

Сочетание ЭС, представляющих системы ИИ, с ИМ позволяет получить качественно новую ступень в создании инструментальных средств проектирования. Если ИМ носят, как указывалось выше, описательный характер, то модели, используемые в ЭС, имеют преобразовательный характер, отражая деятельность специалиста при проектировании. Поэтому задачи, решаемые традиционно на ИМ с помощью специалиста, могут решаться в ЭС без его участия.

В отличие от знаний, полученных от экспертов или другим путем, ИМ является способом хранения и получения, в пределе неограниченных знаний об объекте. Это 
связано с возможностью проведения на модели различных экспериментов и получением при этом новых знаний для заданных условий.

Возможен ряд вариантов взаимодействия ЭС и системы моделирования. В частности обычная ЭС может играть роль интеллектуального интерфейса, позволяющего пользователю выходить на ИМ и методы оптимизации $[11,15]$. При этом гибридная система (ГС) реализует функции не только интеллектуального интерфейса, но и интеллектуального вычислителя (рисунок 3).

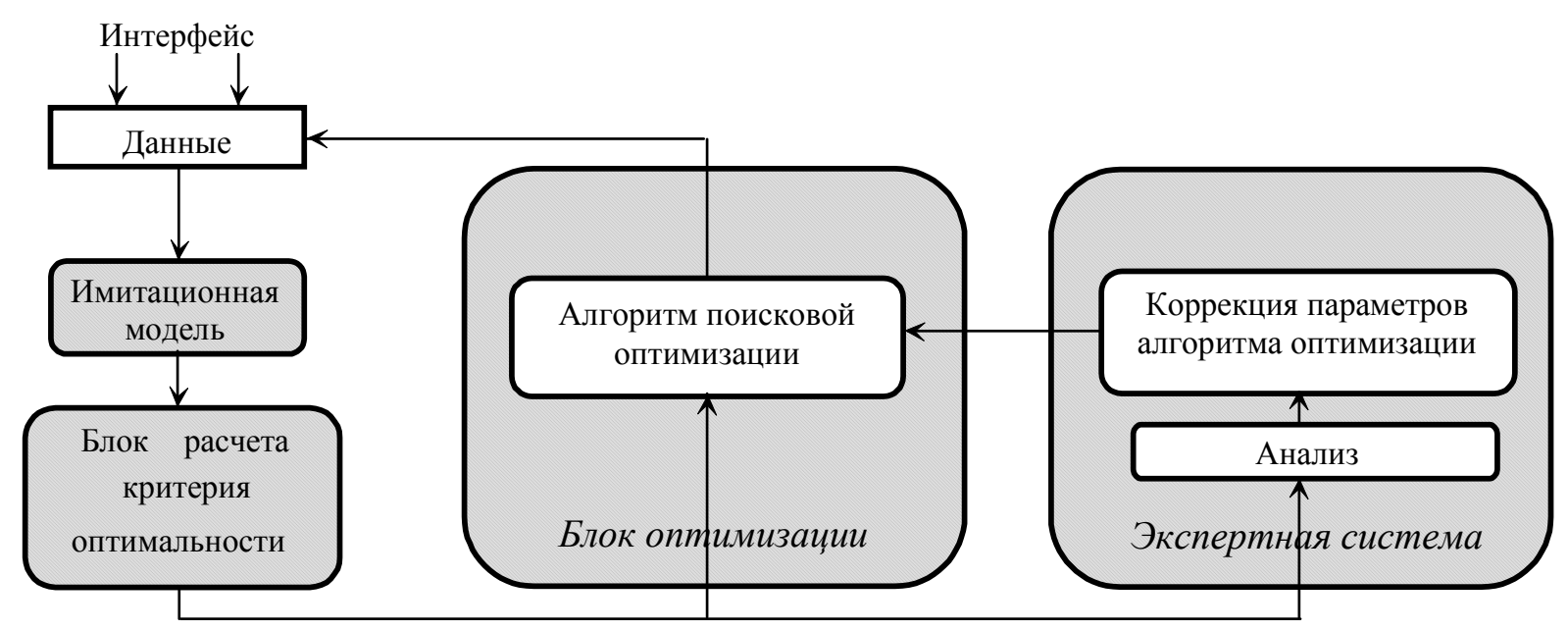

Рисунок 3 - Структура типовой ГС

Рассмотрим функционирование отдельных составляющих такой системы на примере планирования работы некоторого сложного объекта [10]:

- ИМ, служит для составления плана и использует для этого набор эвристических приоритетных правил, для определения приоритета того или иного заказа включаемого в план работ. Чем выше приоритет, тем большим преимуществом по сравнению с остальными пользуется заказ, тем более ранние сроки выполнения он должен иметь в плане.

- Блок оптимизации для подбора приоритетных правил, обеспечивающих получение планов работы с наилучшими показателями. В теории расписаний рассматривается большое количество приоритетных правил, поэтому необходим выбор лучших правил для текущей ситуации, а также выбор оптимальных значений их параметров.

- ЭС, предназначенная для изменения параметров поиска, на основе некоторых представлений человека-оператора о перспективности той или иной стратегии поиска.

Ситуации, в которых необходимо принимать некоторые решения по включению заказа в план называются точками решения. В ИМ рассматриваются следующие точки решения:

- после того как выполнение некоторой работы по выполнению заказа завершилось, необходимо выполнить назначение станка для следующей работы заказа. Если есть только одна возможность, то это сделать просто, но иногда имеется несколько возможностей. В этом случае мы выбираем работу 
заказа, который имеет большее значение приоритета на данном производственном участке из всех остальных заказов.

- когда некоторый производственный участок освободился, мы должны решить какой следующий заказ будет выполняться на станке. В этом случае для каждого заказа, который назначен на обработку на данном станке, но еще не выполнялся, вычисляется приоритет и затем выбирается заказ с максимальным значением приоритета.

Цель блока оптимизации - улучшение решения за счет подбора значений управляемых переменных, при этом достижение действительного оптимума менее важно для таких сложных систем, как рассматриваемая производственная система. Применение точных методов оптимизации, помимо большой сложности, нецелесообразно еще и потому, что алгоритм планирования, который оптимизируется сам по себе эвристический.

Таким образом, гибридная система позволяет использовать сильные стороны различных подходов как математических и вычислительных, так и эвристических, что следует считать типичным для СДС.

\section{Список информационных источников}

[1] Советов Б.Я., Яковлев С.А. Моделирование систем: Учеб. Для вузов -3-е изд., перераб. и доп. // - М.: Высш. шк., 2001. - 343 с.

[2] Ахохов А.Ч. Вложенная имитационная модель транспортировки в условиях стохастического характера грузопотоков и дальности перевозки/ В.И. Нестеренко, А.Ч. Ахохов, А.М.. Ивахненко, А.Б. Чубуков // Вестник МАДИ(ГТУ), вып.1(12) / МАДИ(ГТУ). - М., 2008. - С.70-73.

[3] Jiang, Z., Liu, S., Dougal, R. Design and Testing Spacecraft Power System Using VTB // IEEE Transactions on Aerospace Electronics and Systems. - 2005. Vol. 39, - No. 3. pp. 976-989.

[4] Fishwick P.A. A taxonomy for simulation modeling based on programming language principles // Journal of Computing and Information Science in Engineering March 2001 - Volume 1, - Issue 1, 2001, - pp. 84-91.

[5] .Бенькович Е.С., Колесов Ю.Б., Сениченков Ю.Б. Практическое моделирование динамических систем. - СПб.: БХВ-Петербург, 2002. - 464 с.

[6] Ахохов А.Ч. Методика прогнозирования технико-экономических показателей транспортных предприятий по модели факторного анализа / В.И. Нестеренко, А.Ч. Ахохов, А.М. Ивахненко // Вестник МАДИ(ГТУ), вып.2(13) / МАДИ(ГТУ). M., 2008. - C.74-77.

[7] Марка Д.А., Мак-Гоуэн К. Методология структурного анализа и проектирования. М., Метотехнология, 1993. - 240 с.

[8] Borutzky W. Bond Graphs a methodology for modelling multidisciplinary dynamic systems, SCS, 2004. -440 p.

[9] Коткин Г. Л., Черкасский В. С. Компьютерное моделирование физических процессов с использованием MATLAB: Учеб. пособие / Новосиб. ун-т. Новосибирск, 2001. - 173 с.

[10] Могилевский В.Д., Формализация динамических систем. - М.: Вузовская книга, 1999. - 216 c.. 
[11] Николаев А.Б., Брыль Н.В., Кузнецов С.А. Теоретические основы решения информационно сложных задач / // Вестник МАДИ, вып. 4(23). - М., 2010. - С.7478.

[12] Остроух А.В. Исследование начального периода моделирования на точность среднеинтегральной оценки имитационных моделей / А.В. Остроух, А.А. Солнцев, Н.В. Солдатов, К.А. Новицкий, П.С. Якунин // Вестник МАДИ. - 2010. Вып. 2(21). - С. 61-65.

[13] Остроух А.В. Основы построения систем искусственного интеллекта для промышленных и строительных предприятий: монография / А.В. Остроух. - М.: OОO «Техполиграфцентр», 2008. - 280 с. - ISBN 978-5-94385-033-2.

[14] Остроух А.В. Системы искусственного интеллекта в промышленности, робототехнике и транспортном комплексе: монография / A.В. Остроух Красноярск: Научно-инновационный центр, 2013. - 326 с. - ISBN 978-5-90631410-9.

[15] Остроух А.В. Информационные технологии в научной и производственной деятельности / [ред. А.В. Остроух] - М: ООО "Техполиграфцентр", 2011. - 240 с. ISBN 978-5-94385-056-1. 